\title{
El aprendizaje cooperativo en la universidad. valoración de los estudiantes respecto a su potencialidad para desarrollar competencias
}

Cooperative Learning at University. Assessment of Students Regarding its Potential to Develop Skills

\author{
María Rosa García-Ruiz \\ Universidad de Cantabria \\ rosa.garcia@unican.es \\ Natalia González Fernández \\ Universidad de Cantabria \\ gonzalen@unican.es
}

\section{Resumen}

Se presentan los resultados de la implementación de las metodologías activas, concretamente del aprendizaje cooperativo y trabajo por proyecto, y de la adquisición de competencias que genera en el alumnado, como enfoque innovador en la educación; contextualizado en una facultad de educación

Para ello se ha diseñado un cuestionario, con el que son los propios estudiantes los que valoran su aprendizaje siguiendo esta metodología, respecto a los métodos de carácter más tradicional que se ha venido utilizando en la docencia universitaria, basada, fundamentalmente, en la lección magistral.

Se observa, tras el análisis de los datos, cómo la valoración del alumnado en la consecución de estas competencias es significativamente más positiva tras la implementación del aprendizaje 
cooperativo por el profesorado, a pesar, incluso, de que el tiempo de dedicación a los trabajos grupales se incremente considerablemente.

Como conclusión destacamos que los estudiantes consideran que no solo aprenden más, sino que su aprendizaje es de más calidad con esta metodología, desarrollando una serie de competencias esenciales en la formación universitaria y muy valiosa también para el ámbito profesional.

PALABRAS CLAVE: Metodologías Activas, Aprendizaje cooperativo, competencias, trabajo por proyectos

\section{Abstract}

We present the results of implementing active methodologies, specifically cooperative learning and project work, and the acquisition of skills in students generated as innovative approach in education contextualized in a faculty of education For this we have designed a questionnaire, with which is the students themselves who value their learning following this methodology, compared to more traditional methods that have been used in university teaching, primarily based on lectures. Is observed after the analysis of the data, how the assessment of students in achieving these competencies is significantly more positive after the implementation of cooperative learning by teachers, although even that the time devoted to group work will increase significantly.

KEY WORDS: Active Methodologies, Cooperative Learning, Skills, Project-based Learning.

Fecha recepción: Marzo $2013 \quad$ Fecha aceptación: Junio 2013 


\section{Introducción}

El profesor universitario del Siglo XXI debe ser un profesional competente, al menos en cuanto a las tres funciones que debe desarrollar: la docencia, la investigación y la gestión.

A pesar de que la actividad docente del profesorado universitario no está suficientemente valorada, sino que se considera una función secundaria, frente a la investigadora, que se premia no sólo económicamente, sino con prestigio académico y reconocimiento social; en los últimos años se está reconsiderando la importancia de esta función como condición necesaria para lograr la mejora de la calidad universitaria.

Son numerosos los trabajos publicados que justifican la necesidad y la importancia de la formación metodológica de los docentes en la enseñanza superior, independientemente de la llegada del denominado EEES (De la Cruz, 2003, Tórrego 2004, García y González 2005).

En este sentido, en algunas universidades españolas, entre ellas la Universidad de Cantabria, se están promoviendo iniciativas para potenciar la función docente, tradicionalmente labor de los ICEs, llevando a cabo programas de formación permanente de profesorado, centrados en formación didáctica adecuada a las nuevas competencias que debe adquirir el alumno universitario y al concepto enseñanza-aprendizaje que se desprende del EEES; además de formación en nuevas tecnologías, con una doble intención, por un lado, se pretende conseguir que el profesor domine determinadas técnicas didácticas, y por otro, que pueda enseñar a sus alumnos a utilizarlas y aplicarlas posteriormente en su desarrollo profesional.

La Universidad de Cantabria cuenta, desde el curso 2004/05, dentro del Vicerrectorado de Calidad e Innovación Educativo, con la "Unidad de Apoyo a la Docencia" (http://www.unican.es/Vicerrectorados/calidad_apoyo/apoyodocencia/formacionp). Se trata de un programa destinado a informar al Profesorado de la universidad de las estrategias pedagógicas y tecnológicas necesarias para impulsar el perfeccionamiento del Profesorado, mejorando el rendimiento académico y potenciando la innovación educativa. 
La formación pedagógica ofertada se centra fundamentalmente en cinco grandes líneas o temáticas: Formación en competencias, Estrategias de aprendizaje, Procedimientos de evaluación, Tutorías y Técnicas de Enseñanza Virtual. Estas temáticas constituyen el área de formación pedagógica del módulo para profesores noveles a los que se les certifica su formación mediante el "Diploma de Innovación Docente UC".

Tomando esta formación pedagógica como marco de referencia de este trabajo, es necesario puntualizar que las autoras, además de haber recibido formación en diferentes cursos, también imparten cursos dentro de los ofertados por la Unidad de Apoyo a la Docencia. Fruto de este interés por la metodología docente universitaria hemos podido implementar diferentes estrategias de enseñanza, aprendizaje y evaluación con nuestros alumnos durante los últimos cinco cursos. Por otro lado, tras haber participado en un proyecto de $1+D+l$ del Ministerio de Educación español (convocatoria 05-08) en el que se recoge la opinión del profesorado de cuatro universidades españolas respecto a diferentes aspectos relacionados con el EEES, hemos conocido cuál es el grado de conocimiento, de uso y de importancia que le otorgan a diversas estrategias metodológicas, el uso que se hace de las TIC, o la importancia que se le concede a la evaluación y a la organización del programa de las asignaturas, como parte de la labor docente (Guerra, González y García-Ruiz, 2010; García, Guerra, González y Álvarez, 2010).

Fruto de nuestras líneas de investigación y de nuestra experiencia docente, queremos hacer una apuesta firme por las metodologías activas como las más adecuadas para la formación de personas adultas, no sólo universitarias. En este sentido, y debido a las características de esta publicación, centraremos nuestro análisis en nuestra experiencia basada en la implementación del Aprendizaje Cooperativo y del trabajo por Proyectos con los alumnos de la titulación de Magisterio (especialidad de Educación Infantil) de la Facultad de Educación de la Universidad de Cantabria, durante los últimos cinco años.

\section{EL APRENDIZAJE COOPERATIVO COMO PROPUESTA METODOLÓGICA}


Tradicionalmente la docencia universitaria ha estado caracterizada por la clase magistral, expositiva y en una sola dirección, en la que el profesor transmite unos contenidos por vía oral, cada vez con mayor frecuencia apoyado por las nuevas tecnologías, y con escasa participación por parte de los alumnos. Sin embargo, esta técnica se sigue utilizando y posiblemente se mantendrá en el nuevo sistema universitario, ya que si se utiliza correctamente y combinada con otras estrategias se pueden obtener los resultados deseados. Por otra parte, muchos expertos están trabajando en el desarrollo y correcta aplicación de nuevas metodologías docentes, que sin duda contribuyen a lograr el éxito de la labor del profesor (Gairín et al.., 2004, García, 2005; Mayor 1998; Medina, 2001; Méndez, 2005; Sánchez 1998, Torre y Gil, 2004; Troiano, 2000; Villar, 2004; Zabalza, 1999), pues parece una concepción asumida por la mayoría que el éxito de la aplicación de la metodología docente radica en la combinación armónica de diferentes estrategias, que han de ser coherentes con los intereses y necesidades de los alumnos.

Desde nuestra forma de entender la docencia, no existe un método de enseñanza óptimo, con el que lograr los mejores resultados, sino que cada profesor debe adaptar su forma de enseñar a las distintas situaciones, a los diferentes grupos de alumnos, a las diferentes materias; en definitiva, deber adaptar su forma de enseñar a la forma de aprender del alumno. Por lo tanto, puesto que existen formas de aprendizaje diferentes, es preciso que el profesor encuentre la mejor forma de comunicar los contenidos fundamentales y de enseñar aquellas competencias previamente establecidas. Coincidimos con De la Cruz (1999 y 2003) cuando afirma que cada método debe ajustarse a diferentes factores para lograr la excelencia, factores como los objetivos que persigue, las características de los alumnos, las exigencias de la asignatura, la personalidad del profesor, las condiciones físicas y materiales del centro y el clima de la clase.

En cuanto al cambio que se requiere del profesorado, creemos conveniente destacar la necesidad de realizar un proceso de reflexión acerca del papel que tradicionalmente ha venido asumiendo el docente. En este proceso de convergencia la actividad docente adquiere nuevos 
enfoques (tutorización y atención más personalizada del alumno, seguimiento y evaluación de las actividades no presenciales, coordinación entre docencia presencial y no presencial...), lo que supone implicaciones directas en la metodología docente (Zabalza, 2000 y 2003; Mayor, 2003). Es por esto por lo que consideramos fundamental el conocimiento y utilización de otras técnicas o estratégicas metodológicas que aseguren el proceso de enseñanza - aprendizaje, en el que el alumno ha de ser el eje fundamental, en torno al cual gire el diseño de los curricula de cada plan de estudios.

Nuestra aportación en esta comunicación pretende presentar el aprendizaje cooperativo como propuesta metodológica adecuada en la formación universitaria, lo cual no quiere decir que se desestimen otras propuestas, como puede ser el Portafolio, el Aprendizaje Basado en Problemas, o la Lección Magistral Participativa, sino que se pueden complementar. Por ello es necesario tener en cuenta las ventajas que supone el Aprendizaje Cooperativo, a partir de Cuseo (1996), para el proceso de enseñanza, aprendizaje y evaluación, frente a otras metodologías:

1. Promueve la implicación activa del estudiante en el proceso de aprendizaje.

2. Capitaliza la capacidad que tienen los grupos para incrementar el nivel de aprendizaje mediante la interacción entre compañeros.

3. Promueve el aprendizaje independiente y autodirigido.

4. Facilita el desarrollo de la habilidad para escribir con claridad.

5. Facilita el desarrollo de la capacidad de comunicación oral.

6. Incrementa la satisfacción de los estudiantes con la experiencia de aprendizaje y promueve actitudes más positivas hacia el material de estudio.

7. Permite la preparación de los estudiantes como ciudadanos.

8. Permite desarrollar la capacidad de liderazgo.

9. Prepara a los estudiantes para el mundo del trabajo actual. 
Johnson y Johnson (1989) señalan que se pueden lograr cinco elementos esenciales: Interdependencia positiva; interacción cara a cara; responsabilidad individual; habilidades sociales y procesamiento grupal autónomo.

En nuestra propuesta metodológica se ha optado por complementar el aprendizaje cooperativo con el trabajo por proyectos como estrategia que dota de sentido a la actuación de los estudiantes a lo largo de la asignatura. Se plantea aprender a partir de la realización de un proyecto, de forma conjunta, en la que se la propuesta se basa en resolver un problema en función de un plan, en el que los estudiantes han de identificar el qué, con quién, para qué, cómo, cuánto, factores de riesgo a tener en cuenta, medidas complementarias para lograr resolverlo, resultados esperados, etc.

El proyecto a realizar consistía en la elaboración de una programación para la etapa de Educación Infantil, también denominada "Proyecto", en el que se recogiesen todos los elementos de un proyecto de trabajo adaptado a esta etapa educativa siguiendo las fases propias de un proyecto: elaboración o propósito, preparación o planificación, ejecución y evaluación (Ibáñez, 2008; Muñoz y Zaragoza, 2011; Parra, 2011 y Vizcaíno, 2008). Todo este proceso de trabajo por proyectos se organizaba en torno a la organización de equipos de trabajo cooperativos, siguiendo las pautas anteriormente expuestas.

Por otro lado, la formación del S. XXI, tanto universitaria como en las etapas obligatorias, se caracteriza por la aparición de un concepto, "las competencias", que si no es nuevo, sí ha supuesto toda una revolución en ambos sistemas educativos, por lo que supone de relevancia para la formación a lo largo de la vida. La educación obligatoria y postobligatoria debe formar personas libres, ciudadanos comprometidos con la sociedad, profesionales competentes en sus profesiones, es decir, no sólo se transmiten valores y conocimientos culturales, sino que se transmiten una serie de valores, de actitudes, de habilidades, necesarias para la vida, más allá del ámbito académico. Todo ello se consigue con la formación en competencias, a la que dedicamos el siguiente apartado. 


\section{LA FORMACIÓN EN COMPETENCIAS EN LA UNIVERSIDAD.}

Continuando con el planteamiento anterior, consideramos que la Universidad ha de formar ciudadanos responsables que formen parte activa de la sociedad. Lo cuál implica que, aunque la formación profesional no ha de constituir el único tipo de formación que se recibe en esta institución, consideramos que es fundamental que la formación en competencias esté adecuada al mercado laboral actual. Por lo tanto, es necesario que exista un flujo continuo de información entre el sistema universitario y el sistema empresarial.

En un trabajo anterior (García, 2008) analizábamos las convergencias y divergencias entre sistema educativo y sistema productivo, en el que destacábamos que a pesar de que la demanda de trabajadores se ve satisfecha por el sistema educativo, sí se evidencia la carencia de formación en algunas habilidades personales, como la capacidad de trabajo en equipo, el liderazgo, la iniciativa, el compromiso, etc.

Son numerosas las fuentes bibliográficas, así como las investigaciones que se están llevando a cabo para detectar esta nueva demanda. A modo de ejemplo podemos citar un estudio de la Universidad de Granada (Martínez, 2002), en el que se destaca que los empresarios echan en falta en la preparación de los universitarios una serie de conocimientos no exactamente académicos, como son la capacidad de integración, la adaptación al cambio, polivalencia, movilidad, disponibilidad, implicación y compromiso con su puesto de trabajo y con la empresa.

Cajide y otros (2002) realizan un estudio para comprobar en qué medida las competencias adquiridas en la Universidad responden a las habilidades requeridas por los empresarios, destacando la carencia en algunas habilidades sociales como el trabajo en equipo, la capacidad de comunicación y saber establecer relaciones. 
También es interesante el trabajo realizado por "La Agencia para la calidad del sistema universitario de Cataluña" (2003), en el que se descubre que los titulados universitarios catalanes "suspenden" en una serie de competencias o habilidades que demandan las empresas, tales como la capacidad de gestión, la capacidad para expresarse, tanto de forma oral como escrita, la capacidad de liderazgo, la toma de decisiones, el pensamiento crítico o la creatividad, además de en otras competencias más instrumentales, como son los idiomas y la informática.

Por último haremos referencia a las competencias más buscadas para el empleo, según la consultoría Seeliger y Conde, especializada en la búsqueda de directivos, entre las que destacan como competencias más demandadas en el entorno empresarial las siguientes:

- Facilidad para ilusionarse por la posición, alegre, proactivo, que sepa transmitir con energía el proyecto que va a desarrollar.

- Liderazgo suficiente para seleccionar, dirigir, motivar y gestionar su equipo, con una clara actitud de preocuparse por los demás y de su mejora como profesionales y como personas.

- Abierto, transparente, fácil de relación a todos los niveles, que destaque por sus habilidades de comunicación.

- Autosuficiente, con capacidad de tomar decisiones por sí mismo.

- Con iniciativa y creatividad para aportar nuevas ideas y sugerencias en la mejora de sus áreas de responsabilidad.

- Con criterio, seriedad, íntegro y honesto.

- Con perfil internacional, manejo de idiomas y disponibilidad para viajar.

- Que sea una persona equilibrada personal, familiar y profesionalmente hablando. 
Según Lobato (2006), las competencias que los estudiantes universitarios desarrollan tras lograr trabajar de forma autónoma con éxito son la competencia para aprender a aprender, competencia en el pensamiento crítico, competencia en automotivarse, competencia para comunicarse de modo eficaz y correcto, competencia en la utilización pertinente de las TIC, competencia en la resolución creativa de problemas y competencia en saber trabajar colaborativamente y cooperativamente.

Del mismo modo, señala Apodaca (2006) que las competencias que desarrollan los estudiantes universitarios a través del trabajo en grupo, y que clasifica como competencias de orden social, serían las relacionadas con estrategias de tarea, como plantear cuestiones, pedir aclaraciones, verificar la comprensión, centrar al grupo en el trabajo, elaborar a partir de ideas de otros, dar información o ideas, seguir consignas, regular en tiempo de trabajo, practicar la escucha activa y resumir. Por otro lado se desarrollan competencias más ligadas a estrategias de relación, como reconocer las aportaciones de los otros, verificar la existencia de consenso, expresar correctamente el desacuerdo, animar a los demás, expresar apoyo, invitar a expresarse, reducir tensiones, mediar en los conflictos, expresar sentimientos y demostrar aprecio.

En cuanto a las competencias que consideramos conveniente desarrollar en los estudios de Magisterio, hemos apostado firmemente por aquellas que no sólo van a ayudar al estudiante a lograr el éxito académico en sus estudios, sino que además, le van a ayudar a desenvolverse de una manera más eficaz y acertada en su posterior carrera profesional, como son la comunicación verbal, la capacidad de síntesis, de análisis y reflexión sobre la información recibida, la crítica constructiva, la implicación en las diferentes tareas a realizar, la capacidad de autonomía y la iniciativa.

Tras la lectura de los datos anteriores, consideramos conveniente plantear nuestra propuesta de formación en competencias mediante el aprendizaje cooperativo y el trabajo por proyectos, la cual se ve avalada por los resultados obtenidos durante estos años y que a continuación presentamos. 


\section{LA FORMACIÓN EN COMPETENCIAS MEDIANTES EL APRENDIZAJE COOPERATIVO Y EL TRABAJO POR PROYECTOS: PRINCIPALES RESULTADOS DESDE EL PUNTO DE VISTA DE LOS ESTUDIANTES.}

Como planteábamos anteriormente, durante los últimos cinco años, hemos venido trabajando con el alumnado de la titulación de la Facultad de Educación de la Universidad de Cantabria, en la asignatura Didáctica de la Educación Infantil; utilizando el Aprendizaje Cooperativo como estrategia metodológica, combinado con el trabajo por proyectos. Como es lógico, a lo largo de los diferentes cursos, la puesta en práctica de esta metodología ha conllevado la realización de cambios en el programa de las asignaturas, en la evaluación, en la utilización de los recursos para el desarrollo de las clases... A pesar de los inconvenientes encontrados, hemos de avanzar ya que los resultados obtenidos, tanto en el rendimiento académico de los estudiantes, como en el clima generado en el aula, o en la relación establecida con el alumnado, han merecido la pena.

De entre las diversas técnicas de Aprendizaje Cooperativo, hemos optado por la aplicación de dos de las más conocidas, el puzzle y el grupo de investigación, las cuáles describimos a continuación:

- Técnica de "puzzle" o "rompecabezas" de Aronson (1978): El profesor divide o fragmenta la materia a estudiar en tantas secciones como miembros compongan el grupo de trabajo. Cada alumno se encargará de leer su texto. Posteriormente se reúnen en "grupos de expertos" con alumnos que han leído el mismo texto, para compartir la información. Finalmente, los alumnos vuelven a sus grupos de origen para elaborar conjuntamente todo el material. 
- Técnica de "Grupo de investigación” de Sharan (1980): Los alumnos escogen subtemas de la unidad o materia que tienen que estudiar en clase. Cada grupo divide los subtemas en tareas individuales dentro del grupo. Los alumnos investigan los subtemas juntos y después presentan los resultados a toda la clase. Esta técnica tuvo su desarrollo en la elaboración del proyecto para la etapa de Educación Infantil, planteado en la asignatura.

Puesto que el objetivo de esta publicación no es abordar el proceso de implementación de estas técnicas, procederemos a presentar los resultados obtenidos tras dicha aplicación. Para obtener estos resultados, se diseñó un cuestionario, a través del cual los estudiantes debían valorar el grado de consecución de determinadas habilidades al finalizar el curso, en función de las técnicas utilizadas. Dicho cuestionario fue adaptado del SEESQ (Students Evaluations of Educational Quality), elaborado por Marsh, H.W. y Roche, L.A. (1970) de la Univerisdad de Westem Sydney MacArthur, Autralia; y del Cuestionario de Competencia Social elaborado por Torbay, Muñoz de Bustillo y Hernandez (2001).

El cuestionario consta de 25 items, en los que lo estudiantes valoran de 1 a 5 de grado de acuerdo con una serie de afirmaciones. Como decíamos, los estudiantes deben valoran la consecución de una serie de habilidades planteadas, tanto mediante la metodología utilizada en la asignatura, como en otras en las que se haya trabajado con otro tipo de técnicas más tradicionales como puede ser la Lección Magistral.

Cada curso, al finalizar las asignaturas los estudiantes cumplimenta el cuestionario, por lo que se han obtenido respuestas de 416 estudiantes, desde el curso 2006/07 hasta el curso 2010/11.

Las habilidades que se plantea trabajar con estos grupos de estudiantes se han propuesto en coherencia con aquellas capacidades o competencias que consideramos imprescindibles en la formación universitaria de grado, y que al mismo tiempo, facilitan la adquisición de otras de carácter más específico que se adquirirían en el postgrado, y de las anteriormente planteadas como demandadas por las empresas a sus trabajadores. Por tanto, las habilidades que se han 
trabajado en el aula y sobre las que los estudiantes manifiestan su opinión en el cuestionario son las siguientes:

- Comunicación Verbal

- Síntesis

- Análisis y reflexión

- Crítica Constructiva

- Implicación

- Autonomía

- Iniciativa

Tras el análisis de datos de las respuestas obtenidas, podemos adelantar cómo año tras año el trabajo realizado y los resultados obtenidos resultan satisfactorios. A continuación presentamos los datos más relevantes:

La Comunicación Verbal se plantea en el aula como una habilidad fundamental en la formación de todo maestro, puesto que la voz pasa a ser su herramienta más preciada. Por lo tanto, se le dedica una atención especial en todas y cada una de las actividades realizadas por los grupos de trabajo, tanto en el aula, como fuera de ella, facilitándoles una serie de estrategias y de conocimientos para mejorarlo, pero sobre todo, ofreciendo oportunidades para ponerlo en práctica.

Si tenemos en cuenta cuál es la trayectoria de los estudiantes que ocupan nuestras aulas, hemos de pensar que el lenguaje, la comunicación oral, se ha visto potenciada y considerada como aspecto fundamental en el currículo de Educación Infantil, pero desgraciadamente, en la escolaridad obligatoria el lenguaje oral se ve relegado a un segundo plano, ganando importancia el lenguaje escrito. También en el Bachillerato y en los Ciclos Formativos se concede mucha más importancia al lenguaje escrito, por lo que nuestro alumnado universitario se ve en ocasiones forzado a realizar exposiciones orales ante sus compañeros, sin ninguna experiencia previa, y 
por supuesto, sin ninguna preparación. En este sentido, Escalona y Loscertales (2005) señalan que los estudiantes consideran su capacidad de comunicación oral como una de las competencias en la que se sienten menos seguros, principalmente en "facilidad de soltura y palabra ante un auditorio" y "conexión con el público".

Por lo tanto, no resulta sorprendente, que algo más del $90 \%$ de los estudiantes que han respondido a nuestro cuestionario considere que ha mejorado su Comunicación Verbal trabajando en grupos cooperativos. También existe un $8 \%$ de los estudiantes que consideran haber desarrollado esta habilidad a través de un aprendizaje más tradicional.

La capacidad de sintetizar la información recibida, o de extraer lo fundamental de lo secundario, es necesario en todo estudiante, independientemente del tipo de metodología que se utilice en el aula. A partir de la experiencia vivida con nuestros estudiantes, su percepción respecto a la consecución de esta capacidad de síntesis nos indica que mejora mediante el trabajo con grupos de aprendizaje cooperativo, según la opinión del 95\% de los estudiantes encuestados. Sin embargo, el $10 \%$ de estos mismos estudiantes también consideran que esa capacidad se puede desarrollar con la enseñanza más tradicional.

Considerando la capacidad de análisis y reflexión como la capacidad para deliberar, pensar, repasar, reconsiderar y madurar una idea antes de tomar una decisión, es necesario tener en cuenta que el hecho de trabajar en grupo y de tener que cumplir unos objetivos previamente establecidos con el profesorado, requiere el desarrollo de esta capacidad de forma individual. El profesor debe ser guía, facilitador, acompañante del aprendizaje de sus estudiantes, lo cuál requiere que sea también quien les haga llegar la información proveniente de diferentes fuentes, para poder realizar las actividades propuestas. Esto supone poner en marcha la capacidad de síntesis, anteriormente presentada, y la capacidad de análisis y posterior reflexión en grupo para realizar el trabajo encomendado de la mejor manera posible. El 92\% de los estudiantes que han trabajado mediante esta metodología opinan que se ha desarrollado más 
su capacidad de análisis y reflexión, frente a un $8 \%$ que consideran que se puede desarrollar también mediante metodologías más tradicionales.

Entendemos por crítica constructiva la capacidad para posicionarse ante las intervenciones de otros, cuando éstas son opuestas o diferentes a las propias, aportando su visión y enriqueciendo el resultado. El trabajo en grupo cooperativo exige necesariamente la contraposición de diversos puntos de vista, de opiniones diferentes, por lo que es imprescindible saber escuchar a los otros, saber exponer las propias ideas de la mejor manera posible y saber negociar y llegar a acuerdos que beneficien a todos. Cada integrante de los grupos ha de tener en cuenta que todas y cada una de las intervenciones han de ser respetadas, por lo que se necesita esta capacidad de crítica constructiva que no menosprecie o anule otras aportaciones. La respuesta de nuestros estudiantes ante esta pregunta nos indica que el 93\% considera que se mejora esta capacidad mediante el aprendizaje cooperativo, aunque también consideran que puede mejorarse a través de otras estrategias de enseñanza más tradicional, según el $17 \%$ de los encuestados.

Todo trabajo en equipo requiere implicación de todos sus miembros para obtener los mejores resultados. Por implicación entendemos la capacidad para aportar sugerencias e ideas en las diferentes actividades, comprometiéndose en su desarrollo hasta el final. Para que los estudiantes se impliquen en las tareas asignadas y en el aprendizaje de las asignaturas es necesario que el profesorado establezca unos objetivos claros, de forma consensuada con sus alumnos. En la metodología de aprendizaje cooperativo, especialmente, se requiere que todos y cada uno de los miembros del grupo se comprometan y se impliquen desde la constitución de los grupos. La labor del profesorado es muy importante en este caso, puesto que en ocasiones la motivación de los estudiantes decrece a lo largo del curso, lo cual conlleva que el grado de implicación disminuya. Esa labor de acompañar a los grupos, de crear un clima social positivo en el aula y de permanente contacto con los grupos es fundamental para mantener el grado de implicación. Nuestros estudiantes manifiestan que la capacidad de implicación se desarrolla mejor mediante el aprendizaje cooperativo (para el 98\%). También la implicación en el 
aprendizaje se puede lograr mediante otras metodologías más tradicionales, para un $15 \%$ de los estudiantes encuestados.

En páginas anteriores hemos abordado el cambio de rol que para el profesorado supone el nuevo sistema universitario. Sin embargo no hemos de olvidar lo que conlleva de cambio para el estudiante. Ese cambio hacia un alumnado activo, responsable, autónomo, que ha de adquirir una serie de competencias socioprofesionales que presentábamos más arriba, requiere un esfuerzo por parte del profesorado, que no sólo ha de cambiar su planteamiento metodológico, sino que ha de lograr que el alumno cambie con él. Nuestra apuesta por el Aprendizaje Cooperativo se ve más reforzada aún cuando podemos comprobar cómo facilita ese cambio, tanto al profesorado como al alumnado. Dicho cambio requiere que el estudiante sea un estudiante autónomo en su proceso de aprendizaje, entendiendo por Autonomía la capacidad para resolver actividades utilizando los recursos propios, sin recurrir a la ayuda inmediata del docente. Escribano (1995) demostraba como Aprendizaje Cooperativo y Autónomo va necesariamente unido en el sistema universitario, y así lo consideran nuestros estudiantes, puesto que algo más del $91 \%$ afirma haber mejorado esta capacidad con la metodología cooperativa. Sin embargo, también el $30 \%$ de ellos considera que se puede mejorar esta capacidad mediante métodos más tradicionales.

El estudiante universitario ha de desarrollar la capacidad de Iniciativa, si es que realmente es un estudiante autónomo y activo. La iniciativa es considerada como la capacidad para iniciar, realizar y resolver diversas tareas. En nuestro caso, los grupos de trabajo cooperativo requieren que todos sus miembros desarrollen esta capacidad para lograr los mejores resultados de una forma eficaz, y así se les plantea en cada una de las actividades a realizar. El $80 \%$ de los estudiantes considera que esta capacidad se mejora considerablemente con este trabajo grupal. 


\section{Conclusión}

La mejora de la calidad de la docencia universitaria requiere necesariamente un cambio de actitud del profesorado universitario, que se ve facilitado por los programas de formación pedagógica que están desarrollando las universidades en la actualidad. Por otro lado, el proceso de enseñanza, aprendizaje y evaluación que requiere este nuevo sistema universitario debe centrarse en el desarrollo de competencias socioprofesionales que faciliten una formación integral, tal y como demanda la sociedad actual.

Una vez conocidas cuáles son las competencias que las empresas demandan de los titulados universitarios, planteamos las metodologías activas como las más adecuadas para lograrlo, teniendo en cuenta cuáles son los cambios que supone tanto al profesorado como a los estudiantes. Es decir, se requiere un nuevo profesor, más centrado en el aprendizaje del alumnado que en la mera transmisión de contenidos; más pendiente de las dificultades que el alumnado encuentra en ese proceso de aprendizaje que en los resultados de la evaluación de contenidos.

Por tanto el papel del profesor pasa de ser transmisor de conocimiento a facilitador del aprendizaje mediante una enseñanza activa y más participativa. Por parte del alumnado se requiere que sea el responsable de su aprendizaje, en el sentido de tomar iniciativas, de tomar decisiones respecto a su aprendizaje, de involucrarse en el trabajo en equipo con los demás estudiantes, en definitiva, en ser autónomo. Todo ello requiere la puesta en marcha de lo que hemos denominado metodologías activas puesto que coincidimos con Tejedor (2009) cuando afirma que la enseñanza en la universidad es básicamente tradicional, con predominio del método expositivo, y señala además que según los trabajos de García-Valcárcel (1991 y 1992), el modelo "interactivo" (lo que hemos denominado metodologías activas), influye más favorablemente en el aprendizaje de los estudiantes, frente al expositivo.

De entre las diversas metodologías hemos apostado, al igual que León y Latas (2005), por el Aprendizaje Cooperativo como aquella más idónea para formar en esas competencias 
socioprofesionales que los estudiantes deben adquirir. A lo largo de estos años de puesta en práctica con los estudiantes, de ajustes en los programas de las asignaturas, en los horarios, incluso en los espacios en los que se desarrollan, de acierto y desaciertos con diferentes propuestas de actividades, podemos reafirmar nuestra apuesta por el Aprendizaje Cooperativo. El trabajo por proyecto a supuesto también una mayor motivación para los estudiantes, la mejora del trabajo autónomo y responsable, y una mejora en la capacidad de búsqueda de información.

La respuesta que hemos obtenido de nuestros estudiantes a lo largo de estos años demuestra que con esta metodología se consigue mejorar la adquisición de determinadas capacidades básicas en su formación universitaria, como son la comunicación oral, la capacidad de síntesis, de análisis y reflexión, la crítica constructiva, la implicación con el grupo de trabajo, la autonomía para mejorar su aprendizaje y la capacidad para tomar la iniciativa.

Al mismo tiempo hemos podido descubrir una serie de ventajas logradas a través de la implementación del Aprendizaje Cooperativo, como son la mayor implicación del alumnado en el proceso de aprendizaje, el aumento del nivel de aprendizaje mediante el trabajo en grupo cooperativo, la potenciación del aprendizaje independiente y autodirigido, el desarrollo de la habilidad de escribir con claridad y de la comunicación oral, el desarrollo de la capacidad de liderazgo. También hemos podido comprobar el incremento de una mayor satisfacción por parte de los estudiantes o una actitud positiva ante la asignatura, así como la preparación de los estudiantes como ciudadanos y como profesionales del mundo del trabajo.

Finalmente, presentamos algunas propuestas de mejora de la aplicación de esta metodología, fruto del análisis y la reflexión que esta experiencia nos ha permitido. Para ello es necesario superar algunos impedimentos que hemos encontrado en dicha implementación, como es el elevado número de estudiantes matriculados en nuestras asignaturas (aproximadamente 85); la mala disposición del mobiliario en las aulas, con mesas y sillas ancladas al suelo; la 
fragmentación del horario lectivo en sesiones de una hora de clase; la escasa o nula experiencia del alumnado en trabajo en grupo cooperativo, etc.

Para superar estos inconvenientes tomamos como referencia el trabajo de Escribano (1995), en el que propone algunos aspectos para facilitar la enseñanza en pequeños grupos cooperativos, a partir del cual hemos adaptado nuestra propuesta metodológica para lograr una mayor eficacia:

1. Ordenar los asientos, de manera que los grupos puedan situarse en círculo. En nuestro caso esto no es posible puesto que las mesas y sillas si no están ancladas al suelo, son tal cantidad que resulta muy complicado moverlas, ya que no hay espacio libre en las aulas. El cambio que hemos realizado ha sido solicitar el uso de dos aulas contiguas para que los alumnos dispongan de espacio suficiente, y al mismo tiempo el profesorado puede desplazarse de una a otra durante las sesiones de trabajo grupal.

2. Organización de los tiempos lectivos en sesiones de más de 3 horas. Consideramos necesario contar con el tiempo suficiente para facilitar la reflexión y el debate posterior a las diferentes actividades realizadas en el aula. En nuestra facultad la tradición establece sesiones de clase de una hora de duración, aunque hemos logrado agruparlas de manera que todos los grupos tuviesen al menos una sesión de dos horas consecutivas semanalmente.

3. Expectativas y reglas básicas a establecer al inicio del trabajo. Como decíamos anteriormente, nuestros alumnos tienen poca experiencia en trabajo cooperativo, aunque sí de trabajo en grupo, en el que cada miembro se asigna una tarea que finalmente se entrega al docente. Para mejorar esta situación se plantean una serie de reglas a tener en cuenta por los integrantes de los grupos, y se exponen claramente cuáles son los objetivos a conseguir en el curso, de manera que todos los estudiantes lo contrasten con sus expectativas y valoren si merece la pena el esfuerzo.

4. Seguridad, creando un clima social positivo en el aula. El clima del grupo clase y su relación con el profesorado es fundamental en esta metodología. Para mejorarlo hemos optado por permitir la formación de los grupos por los mismos estudiantes, aunque sí se 
han forzado algunas ocasiones de admisión de nuevos integrantes. Es importante que los estudiantes se sientan seguros con sus compañeros y con el profesorado, por lo que se ha tratado de ampliar el horario de tutorías y el contenido de estas tutorías.

5. Número de personas para la composición de pequeños grupos (entre 3 y 5, siempre impares). También comentábamos anteriormente que contamos con números de alumnos muy numerosos, por lo que hemos optado por crear grupos de 5 a 7 integrantes.

6. Cooperación, frente a competición o individualismo. Este es uno de los aspectos más importante para garantizar el éxito de esta metodología, y sobre todo para asegurar la formación en las competencias socioprofesionales ya mencionadas. Es fundamental adaptar el trabajo en las aulas universitarias al trabajo como profesionales en la empresa, por lo que el trabajo en equipo, la cooperación, ha de reforzarse y por lo tanto, es necesario ofrecer al alumnado el mayor número de experiencias posible.

Para finalizar con este trabajo creemos conveniente eludir a Delors y destacar que nuestro propósito fundamental es ayudar a nuestros estudiantes a saber, a conocer, a saber hacer, a saber ser y sobre todo, a saber trabajar en equipo.

Y en nuestro empeño por mejorar cada día, continuamos llevando al aula diversas estrategias docentes, que combinadas con el aprendizaje cooperativo, garanticen el éxito no solo del resultado académico de los estudiantes, sino del proceso de enseñanza y aprendizaje, convirtiendo al estudiante en el verdadero protagonista de su aprendizaje.

\section{Bibliografía}

Agencia para la calidad del sistema universitario de Cataluña (2003). Educación Superior y trabajo de Cataluña. Barcelona: AQU.

Apocada, P. (2006). Estudio y trabajo en grupo. Metodologías de enseñanza y aprendizaje para el desarrollo de competencias. Orientaciones para el profesorado universitario ante el Espacio Europeo de Educación Superior. Madrid: Alianza Editorial. 
Aronson, E. (1978). The Jigsaw Classroon. Beverly Hills: Sage Publ.

Cajide, J. et al. (2002). Competencias adquiridas en la Universidad y habilidades requeridas por los empresarios. Revista de Investigación Educativa, 20 (2), 449-467.

Cuseo, J.B. (1996). Cooperative learning: A pedagogy for Addressing Contemporary Challanges \& Critical Issues in Higher Education.Marymount College, New Forums Press.

De la Cruz, A. (1999). Formación del profesor universitario en metodología docente. Aprender y enseñar en la Universidad. Iniciación a la docencia universitaria. Jaén: Universidad de Jaén.

De la Cruz, A. (2003). El proceso de convergencia europeo: Ocasión para modernizar la universidad española si se produce un cambio de mentalidad en gestores, profesores y estudiantes. Aula Abierta, 82, 191-216.

Escalona, A. I. \& Loscertales, B. (2005). Actividades para la enseñanza y el aprendizaje de competencias genéricas en el marco del Espacio Europeo de Educación Superior. Zaragoza: Servicio de publicaciones de la Universidad de Zaragoza.

Escribano, A. (1995). Aprendizaje cooperativo y autónomo en la enseñanza universitaria. Enseñanza, 13, 89-102.

Gairín, J. et al. (2004). La tutoría académica en el escenario europeo de Educación Superior. Revista Interuniversitaria de Formación del Profesorado, 18 (1), 61-77.

García, R. (2005). El cambio metodológico en los docentes universitarios europeos: del método tradicional al aprendizaje cooperativo. Congreso Internacional ¿Hacia dónde va la educación universitaria americana y europea? León: Universidad de León.

García, R. (2008). Convergencias y divergencias entre sistema productivo y sistema educativo. Revista Complutense de Educación, 19 (1), 209-223.

Guerra, S., González, N., \& García-Ruiz, R. (2010). Utilización de las TIC por el profesorado universitario como recurso didáctico. Comunicar, 35, 141-148. 
Ibánez, C. (2008). El proyecto de Educación Infantil y su práctica en el aula. Madrid: La Muralla. Johson, D.W. \& Johnson, R.T. (1989). Cooperative Learning: What Special Education Teachers Needs to Know. Pointer, 33 (2), 5-10.

Lobato, C. (2006). Estudio y trabajo autónomos del estudiante. Metodologías de enseñanza y aprendizaje para el desarrollo de competencias. Orientaciones para el profesorado universitario ante el Espacio Europeo de Educación Superior. Madrid: Alianza Editorial.

Martínez, R. (2002). La inserción laboral de los jóvenes universitarios. Granada: Universidad de Granada.

Mayor, C.M. (1998). El reto de la formación docente en la institución universitaria: un análisis sobre la perspectiva de los profesores principiantes. V Congreso Interuniversitario de Organización de Instituciones Educativas. Madrid, Universidad Complutense y UNED.

Mayor, C.M. (2003). Enseñanza y aprendizaje en la Educación Superior. Barcelona: OctaedroEUB.

Méndez, C. (2005). La implantación del sistema de créditos europeo como una oportunidad para la innovación y la mejora de los procedimientos de enseñanza- aprendizaje en la Universidad. Revista Española de Pedagogía, 230, 5-16.

Muñoz, C. \& Zaragoza, C. (2011). Didáctica de la Educación Infantil. Barcelona: Altamar.

Parra. J.M. (2011). Manual de Didáctica de la Educación Infantil. Madrid: Ibergaceta.

Sharan, S. (1980). Cooperative Learning in teams: recent methods and effects on archievement attitudes and athic relations. Review of Educational Research, 50, 241-271.

Tejedor, F. J. (2009). Evaluación del profesorado universitario: enfoque metodológico y algunas aportaciones de la investigación. Estudios sobre Educación, 16, 79-102.

Tórrego, L. (2004). Ser profesor universitario, ¿un reto en el contexto de convergencia europea? Un recorrido por declaraciones y comunicados. Revista Interuniversitaria de Formación del 
Profesorado, 1 (3), 259-268.

Troiano, H. (2000). Estrategias para el cambio de las prácticas docentes en la universidad. Educar, 27, 137-149.

Torre, J.C. y Gil, E. (2004). Hacia una enseñanza universitaria centrada en el aprendizaje. Madrid: Universidad Pontificia de Comillas.

Villar, L.M. (2004). Programa para la mejora de la docencia universitaria. Madrid: PearsonPrentice Hall.

Zabalza, M.A. (2000). La enseñanza universitaria: el escenario y los protagonistas. Madrid: Narcea.

Zabalza, M.A. (2003). Competencias docentes del profesorado universitario: calidad y desarrollo profesional. Madrid: Narcea. 